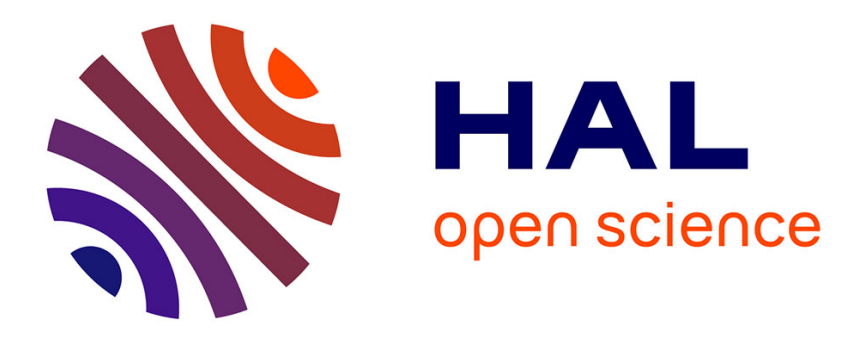

\title{
Réalisation et étude des propriétés de détecteurs de particules lourdes à barrière de surface
}

A. Coche, P. Siffert

\section{To cite this version:}

A. Coche, P. Siffert. Réalisation et étude des propriétés de détecteurs de particules lourdes à barrière de surface. J. Phys. Phys. Appl., 1961, 22 (S11), pp.162-164. 10.1051/jphysap:019610022011016201. jpa-00212840

\section{HAL Id: jpa-00212840 https://hal.science/jpa-00212840}

Submitted on 1 Jan 1961

HAL is a multi-disciplinary open access archive for the deposit and dissemination of scientific research documents, whether they are published or not. The documents may come from teaching and research institutions in France or abroad, or from public or private research centers.
L'archive ouverte pluridisciplinaire HAL, est destinée au dépôt et à la diffusion de documents scientifiques de niveau recherche, publiés ou non, émanant des établissements d'enseignement et de recherche français ou étrangers, des laboratoires publics ou privés. 
LE JOURNAL DE PHYSIQUE ET LE RADIUM PHYSIQUE APPLIQUÉE
SUPPLÉMENT AU N $\mathrm{N}^{0} 11$. TOME 22, NOVEMBRE 1961, PAGE 162 A.

\title{
RÉALISATION ET ÉTUDE DES PROPRIÉTÉS DE DÉTECTEURS DE PARTICULES LOURDES A BARRIËE DE SURFACE
}

\author{
Par A. COCHE et P. SIFFERT,
}

Département de Chimie Nucléaire, Centre de Recherches Nucléaires de Strasbourg.

\begin{abstract}
Résumé. - On a réalisé des détecteurs de particules lourdes à barrière de surface Au-Si. La méthode de fabrication est décrite et l'influence d'un certain nombre de facteurs susceptibles de modifier les propriétés du détecteur est étudiée. On a obtenu une largeur à mi-hauteur de la raie alpha de ${ }^{210} \mathrm{Po}$ inférieure à $30 \mathrm{keV}$ pour une aire utile de l'ordre de $7 \mathrm{~mm}^{2}$. On donne les principales caractéristiques : courant inverse, résolution, vieillissement, modifications entraînées par une forte irradiation alpha...
\end{abstract}

Abstract. - Gold-silicon surface barrier counters for heavy particles have been built. The method of construction is described and the influence of a number of factors which could change the properties of the detector is studied. Full width at half maximum (FWHM) of ${ }^{210} \mathrm{Po}$ alpha rays has been obtained below $30 \mathrm{keV}$ for a sensitive area of $7 \mathrm{~mm}^{2}$. The principal characteristics are given : reverse current, resolution, linearity, radiation damage induced by alpha particles...

Depuis 1959 environ, la détection des rayonnements nucléaires et surtout des particules lourdes (rayons $\alpha$, protons, fragments de fission, ions lourds, ...) à l'aide de semi-conducteurs a fait l'objet d'un grand nombre de publications (une bibliographie complète peut être trouvée dans les références [1] et [2]). Los compteurs utilisés à l'heure actuelle, qui sont de deux types, à barrière de surface, ou à jonction $p-n$, sont constitués essentiellement par une diode que l'on polarise dans le sens bloquant. Si:une particule lourde crée des paires électron-trou dans la région de charge d'espace où règne un champ électrique élevé, il apparaît dans le circuit extérieur une impulsion de tension de hauteur égale à $2 \mathrm{mV} / \mathrm{MeV}$ environ (pour des rayonnements $\alpha$ ); elle est proportionnelle à l'énergie de la particule, si cette dernière a été complètement arrêtée dans cette zone.

Nous avons réalisé des détecteurs à barrière de surface Au-Si dont nous nous proposons de décrire iciles principes de fabrication et les caractéristiques essentielles.

Le matériau de base est constitué par du silicium $\mathrm{n}$ de diverses provenances et de résistivités comprises entre 20 et $200 \Omega-\mathrm{cm}$. Il est utilisé sous forme de pavés carrés ou de disques d'épaisseurs variant entre 300 et $1000 \mu$. Ces pastilles sont d'abord passées à l'émeri fin (environ $4 \mu$ ) pour enlever les traces laissées par la scie servant au découpage, puis dégraissées et enfin lavées très soigneusement à l'eau désionisée. La surface du silicium subit ensuite une attaque chimique dans 
une solution (dite "d'etching ", type C P 4) couramment employée dans la technologie des semiconducteurs. Après lavage on réalise un contact ohmique sur une des surfaces de la plaquette. La surface opposée est alors attaquée, à nouveau, avec la solution C P 4, avant d'y déposer par vaporisation sous vide une mince couche d'or (épaisseur de l'ordre de $500 \AA$ ). La liaison électrique du circuit extérieur et de la couche d'or est obtenue par pression d'un fil de bronze ou d'or.

Différents procédés de formation du contact ohmique ont été essayés. On peut : effectuer, par dépôt spontané, un nickelage du pavé de silicium, puis dissoudre la couche de nickel, sauf sur une face protégée par un revêtement plastique; employer des ciments conducteurs; vaporiser un métal sur la face convenablement traitée.

En définitive, la solution retenue pour les détecteurs dont il est question ici, consiste à souder le pavé, à l'aide d'un alliage étain-antimoine, sur une lame de tantale, portée pendant quelques secondes, sous vide, à $950^{\circ} \mathrm{C}$ environ. Bien que le silicium soit soumis temporairement à une élévation de température importante, cette méthode donne de bons résultats (les procédés de traitement à froid mentionnés ci-dessus, semblent conduire à des détecteurs à courant inverse plus faible, mais de résistance dans le sens direct, plus grande).

De nombreux essais ont mis en évidence l'importance de l'opération d'attaque (effectuée, dans nos expériences, à la température ordinaire) par la solution C P 4: on a fait varier, en particulier sa durée, ainsi que la composition et "l'âge " de la solution. Cette dernière, constituée d'acide fluorhydrique (3 volumes), d'acide nitrique (5 volumes) et d'acide acétique (3 volumes) permet d'obtenir un état de surface convenable, si elle a été préparée environ une heure avant l'attaque et si l'acide nitrique est concentré, $(d>1,38)$. La durée de l'opération dépend de la concentration des acides employés (fluorhydrique et nitrique) mais est, dans les divers cas, comprise entre 3 et 12 minutes. On a intérêt à ne pas utiliser de solutions préparées plus de 24 heures à l'avance.

Dans un certain nombre d'expériences, nous avons remplacé l'or par d'autres métaux : de bons détecteurs ont été, en particulier, réalisés avec une couche d'argent, mais leurs propriétés s'altèrent assez rapidement avec le temps.

La caractéristique inverse de la diode donne généralement une indication sur la qualité du détecteur, d'autant meilleur que le courant inverse est plus stable et plus faible. Pour nos compteurs, celui-ci diminue considérablement à partir de la fin de la fabrication (évaporation de la couche d'or). Cette évolution qui peut se poursuivre pendant plusieurs jours, dépend du milieu dans lequel elle se produit (atmosphère sèche, humide, oxydante) et met en évidence la formation progressive d'une couche d'inversion. Les meilleures diodes ont été obtenues dans une atmosphère d'humidité relative voisine de $70 \%$. Pour des surfaces utiles de $7 \mathrm{~mm}^{2}$, les courants inverses sont inférieurs à $0,5 \mu \mathrm{A}$ pour des tensions de 50 volts (sans protection contre les courants de surface). Les courbes de

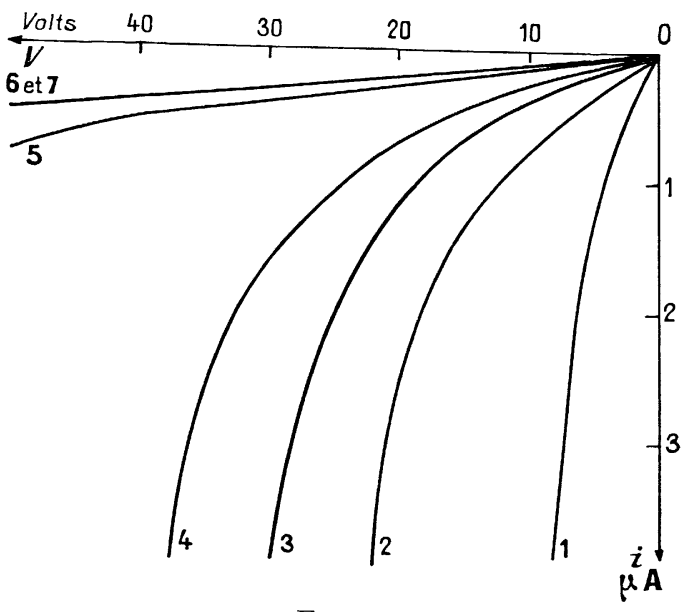

FIG. 1.

1 : 5 minutes après réalisation ; 2 : après 1 heure ; 3 : après 2 heures ; 4 : après 3 heures ; 5 : après 16 heures; 6 : après 18 heures ; 7 : après 3 jours.

la figure 1 montrent les variations que peut subir le courant inverse. Celui-ci devient stable au bout d'un certain temps et n'est alors pas modifié notablement quand le compteur est soumis à un vide poussé. Il n'en est pas de même pour un détecteur qui n'a pas eu le temps d'évoluer et dont les caractéristiques changent sensiblement, comme cela res-

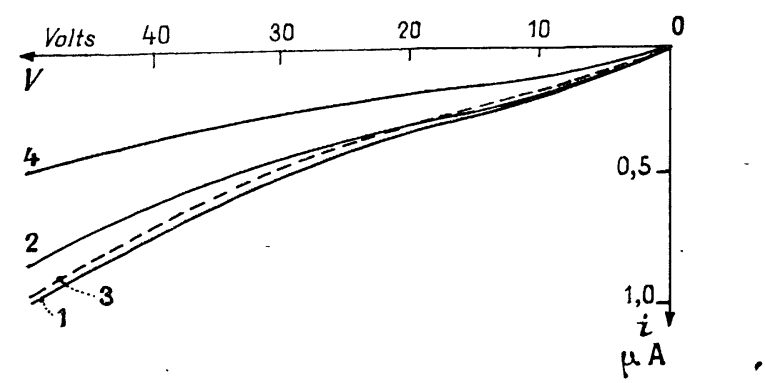

FIG. 2.

1 : à l'air ; 2 : sous vide ; $3: 1$ heure après la fin du pompage $; 4: 5$ jours après la fin du pompage.

sort de la figure 2. Ces diodes sont photosensibles et se comportent comme des cellules photovoltaïques.

Nous avons vérifié que la hauteur de l'impulsion délivrée par le compteur varie, en fonction de la polarisation appliquée, suivant une loi en $V^{1 / 2}$, dès que la tension inverse dépasse quelques volts (fig. 3).

La résolution du compteur utilisé comme spec- 
tromètre dépend, entre autres facteurs, du bruit du détecteur et de celui des circuits électroniques associés. En ce qui concerne le premier, l'existence de fluctuations du courant inverse plutôt que sa valeur absolue est le facteur déterminant. De bonnes résolutions peuvent être atteintes avec des détecteurs à courant inverse relativement jmportant, mais bien stable. L'amplificateur à faible bruit

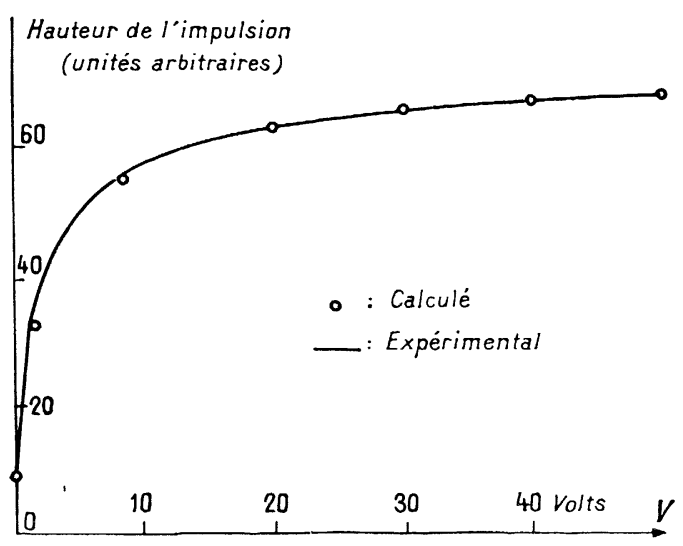

FIG. 3.

associé à la diode, est d'un type voisin de celui décrit par Blankenship [3] (étage d'entrée cascode); sa réalisation a été particulièrement soignée (connexions courtes notamment). Pour le spectre des rayons $\alpha$ de ${ }^{210} \mathrm{Po}$, la largeur à mi-hauteur est inférieure à $30 \mathrm{keV}$, pour des aires utiles de $7 \mathrm{~mm}^{2}$ (dans certains cas, toutefois, nous avons obtenu des valeurs nettement inférieures).

La réponse aux particules $\alpha$ de compteurs réalisés avec du silicium de résistivité $60 \Omega$-cm, est linéaire jusqu'à $9 \mathrm{MeV}$ au moins (limite de l'énergie des particules $\alpha$ dont nous disposions) et ce, pour des tensions inverses de l'ordre de 100 volts. Dans les mêmes conditions, la réponse aux protons cesse d'être linéaire au-dessus de 2,5 MeV.
La stabilité de ces détecteurs a, enfin, été examinée : s'ils sont conservés en atmosphère normale, aucune modification des caractéristiques électriques n'apparait au bout de plusieurs mois. Nous avons d'autre part, constaté qu'une irradiation pendant 140 jours aux particules $\alpha$ d'une source de ${ }^{210} \mathrm{Po}$ de faible intensité (flux intégré de l'ordre de $10^{11}$ ) entraîne une diminution de la résolution

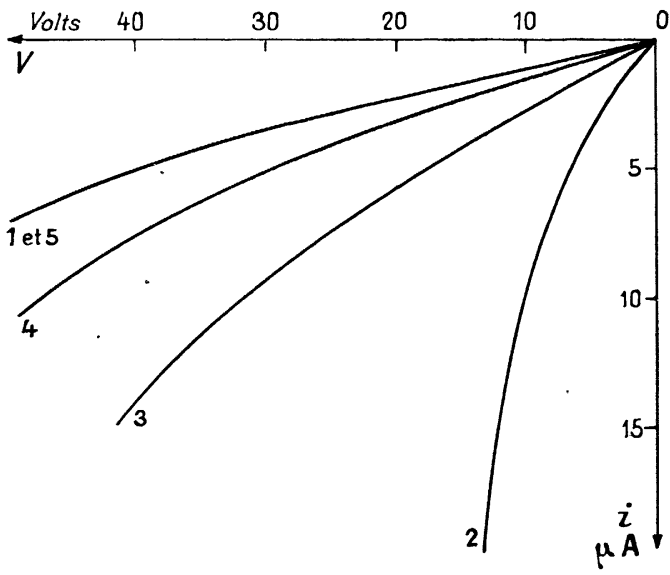

FIG. 4.

$1:$ avant irradiation $; 2:$ après irradiation $; 3: 15$ minutes après fin irradiation ; $4: 24$ heures après fin irradiation ; $5: 1$ semaine après fin irradiation.

dans un rapport 3. Par contre, une forte irradiation de courte durée (environ $10^{11}$ rayons $\alpha$ de ${ }^{210} \mathrm{Po}$ en 5 minutes) provoque la destruction du compteur : le courant inverse, après une augmentation, revient à sa valeur initiale au bout de quelques jours, mais la résolution aux particules $\alpha$ reste définitivement mauvaise (fig. 4).

D'autres expériences sont en cours pour réaliser des détecteurs à région de charge d'espace beaucoup plus profonde, afin d'augmenter le domaine de linéarité et de détecter des rayonnements de faible ionisation spécifique. Nous remercions M. J. Kurek qui a participé à cette étude.

\section{BIBLIOGRAPHIE}

[1] O. R. N. L. 2583 : Bibliography on semi-conductor nuclear radiation detectors, US. Department of Commerce, Washington $25 \mathrm{D}$. C.
[2] I. R. E. Trans. on Nucl. Science, 1961, NS8, no 1.

[3] Blankenship (J. L.) et Borkowski (G. J.), I. R. E. Trans. on Nucl. Science, 1961, NS8, no 1, 17. 